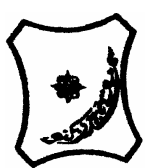

Bayero Journal of Pure and Applied Sciences, 8(2): 46 - 57

Received: August, 2014

Accepted: August, 2015

ISSN $2006-6996$

\title{
POST CHRONIC CIGARETTE SMOKE EXPOSURE AND ASSESSMENT OF LEARNING, MEMORY AND ASYMPTOMATIC LEVEL OF PERFORMANCE IN WISTAR RATS
}

\author{
${ }^{1}$ Badamasi,I. M. and ${ }^{2}$ Lawal, I. U. \\ ${ }^{1}$ Department of Anatomy, Faculty of Basic Medical Sciences, Bayero University Kano, Kano Nigeria. \\ ${ }^{2}$ Department of Physiotherapy,Faculty of Medicine, Bayero University Kano,Kano Nigeria \\ *Correspondence : Email:badamasi_mohammedibrahim@yahoo.com. Phone: 08037024135
}

\section{ABSTRACT}

This study is to further assess the effect of cessation of chronic cigarette smoke exposure(CSE) on memory, learning and asymptomatic level of performance(ALOP). The design of the study was a single blind randomized control trial involving exposed and control group of rats. Water-maze assessment of learning, memory and ALOP revealed a progressive decline in the latency-time with each sessions, for all study groups- implying that learning was taking place. The female exposed rats had the most difficulty in learning the task compared to the other study groups. In-addition, ALOP was averagely attained by the 4th and 5th session for the exposed and control groups. Furthermore, memory for these sessions was demonstrated by all study groups, with the femaleexposed group depicting the highest level. Hippocampal histology revealed a dichotomy between exposed and control groups. The exposed groups had nuclei condensation-pyknosis and edematous changes. Hence, the observation of both a difficulty in learning and a high level of memorization in the female-exposed group suggest that, this study setting favors the recovery of the apparatusesespecially hippocampus, involved in these function from any deleterious effect of chronic CSE. And that the recovery of the males is faster than those of the females.

Key word: psychology, learning, memory retention, Wistar rat, Smoking.

\section{INTRODUCTION}

"Learning is the process through which emerging information about the environment and universe are acknowledged. And memory is the process through which these information are retained" (Lynch, 2004).Assessment of learning and/or memory has consistently been carried out in the animal subjects using various methods, including the ability to find a hidden platform in a tank of water, Morris water maze (MWM) task (Brandeis et al., 1989). The task essentially utilizes training session for the ability of the rats to swim to a hidden-platform as a pointer to learning. Furthermore, a well- learned rat that can swim to the target quadrant and stay in the quadrant for considerable time, even when the platform is removed is said to possess good memory for the target location, a probe trial assessment (Brandeis et al., 1989). There are numerous benefits in using the MWM task for assessing learning and memory especially like it can be accomplished in a short period of time with a relatively modest number of animals. Furthermore, through the use of "training" as well as "probe" trials, learning as well as retrieval processes (spatial bias) or memory can be analyzed and compared between groups (Morris, 1984). This assessment of spatial memory are well established test of hippocampus dependant memory (Toumane and Durkin, 1993). In addtition, defects in spatial memory tasks has been demonstrated following hippocampal damage (Toumane and Durkin, 1993). In-vitro experiments involving the exposure of hippocampal slices to 150 part per million (PPM) of carbon monoxide (CO), revealed that these slices lost their ability to maintain long term potentiation (LTP) (Giampaoloet al., 2000). LTP is known as the basis of synaptic plasticity, which is the electrophysiological means of memory formation (Bliss and Collingridge, 1993). Carbon monoxide (CO) is one of the numerous constituents of cigarette smoke and hence suggesting that there is a relationship between hippocampus, CO, LTP and also hippocampus-dependent task like memory, which might either be positive or negative. A recent study had revealed that cigarette smoke exposure causes hippocampal damage by apoptosis(Eser, et al., 2007).In a study investigating the neuroprotective effect of caffeic acid phenethyl ester (CAPE) in the hippocampal formation, study findingssuggest that cigarette smoking induces 
apoptosis in the hippocampal formation of rabbits and CAPE has a protective role against such induction.

However, the in vivo - functional effects of CSE on learning and memory are not represented by an allor-non statements in the literature as the in vitro studies seems to suggest. Rather, some studies in the literature revealed that cigarette smoke exposure (CSE) was associated with deterioration of learning and memory abilities (Yoltonet al, 2005; Cervillaet al, 2000; Kalmijnet al, 2002; Nowakowskaet al, 2006; Liu et al, 2013). While there are other studies that suggest that CSE was beneficial to learning and memory (Elwood et al, 1999; Ford et al, 1996; Schinkaet al, 2003). Furthermore there are few studies that revealed that cessation of CSE improves learning and memory (Galaniset al, 1997; Fried et al, 2006). While, some other studies revealed that cessation of CSE is detrimental to memory development (Ernst et al, 2001; Adrianna et al, 2005). These contrasting data regarding both the effect of CSE and those of cessation of CSE are probably due to differences in design of these experimental studies and also differences in outcome measures for these studies.

The experimental design of Cigarette smoke exposure studies are compounded by the fact that the dose and duration of exposure for a smoker- the primary smoker, differs significantly from those of other primary smokers and other individuals around the primary smoker- secondary smoker (Fried et al, 2006). Furthermore, someCSE studies in the literature were characterized with exposure to 4-15 ppm of carbon monoxide (Alvin and Terry, 2009; Slotkinet al, 2006). However, such common low dose assessments may not truly represent both the CSE in a primary smoker and those observed in chronic toxic level of exposures. The aim of this current study was to assess learning, memory and asymptomatic level of performance (ALOP) during a prolonged period of cessation of any CSE, in Wistar rats that had previously been exposed to cigarette smoke for a long time.

\section{MATERIALS AND METHODS \\ Ethical consideration}

This study protocol was approved by the animal research ethics committee of Faculty of Medicine, Ahmadu Bello Univerity Zaria Nigeria.

\section{Subjects}

A total of 24 Wistar rats aged 20-24 weeks and weighting between $300-350 \mathrm{~g}$ were randomized into two groups (i.e. exposure and control groups of rats). Each group of rats was made up of 12 rats, with a matched male to female ratio. The rats were housed according to gender, males in different cages from females and allowed normal environmental cycle of 12-hour light or day and night or dark cycle. The study-groups were designated control group A and B for male and female rats and exposed group $C$ and $D$ for male and female rats respectively. Water and animal food pellets from Sanders Feed manufacturing company were made available to all groups ad libitum.

\section{Study duration and structure}

This study involved a 13 week intermittent exposure to cigarette smoke for the rats in the exposure group and atmospheric air exposure for the control group. These exposure periods were followed by an 8 week period of water maze trial, during these water maze trials, further exposure to cigarette smoke was discontinued. Hence, the total duration for this study spans 21 weeks.

\section{Experimental protocol}

\section{Cigarette smoke exposure (CSE):}

The exposure setup was designed as a static exposure apparatus. The CSE was conducted in a rectangular plastic container measuring $50 \times 20 \times 20 \mathrm{~cm}^{3}$. The chamber was designed with a glass roof which could be slided away to allow for ventilation. The chamber was fitted with an Amprobe carbon monoxide meter and an ash tray which were carefully placed in the chamber, protected from contact with the rats. A commercially available cigarette stick was lighted and placed on ash tray and allowed to burn and generate smoke which was meticulously monitored by the carbon monoxide meter which was also used to measure the concentration of carbon monoxide generated from the burning cigarette stick in the chamber. The meter displays the value of carbon monoxide on its screen through the glass cover. The glass cover can be slided away to ventilate the chamber once the concentration and time for the exposure of the rats had been achieved. Rats in the exposure groups (groups $C$ and $D$ ) were exposed for alternate cycles of 2 minutes of exposure and ventilation; for a total of 10 minutes at $150-200 \mathrm{ppm}$ of carbon monoxide (Ahmadnia et. al., 2007) while rats in the control groups $A$ and $B$ were only exposed to atmospheric air for the entire session of the experiment.

\section{Confirmation of cigarette smoke inhaltion:}

Nicotine test strip which detects the presence of cotinine ( $\leq 200 \mathrm{ng}$ ), a metabolite of nicotine in the urine. The test strips was used to test the urine samples of the Wistar rats after the CSE sessions to verify that cigarette smoke was actually inhaled, assimilated and metabolized by the rats. The urine sample was obtained from the rats by placing the rats in a sterile, dry plastic bucket and then closely monitoring them for production of urine. A plastic dropper was then used to aspirate some of the urine and applying it to the well on the nicotine test strip directly. The urine is adsorbed through capillary wick action onto the test surface of the strip and the appearance of two (2) pink lines suggest the presence of cotinine in the urine sample.

\section{Morris water maze task:}

Rats in all the groups were subjected to a total of seven (7) sessions of Morris water maze training over two months. Each session involved one (1) Morris water maze training. The sessions were carried out every 7th day of the week for 2 months. The Morris water maze training involved releasing the rats into a Morris water maze tank measuring $120 \mathrm{~cm}$ in diameter and $60 \mathrm{~cm}$ deep filled with water. The water maze tank was fitted with a hidden platform at one of its quadrant. 
The hidden platform was painted black just like the water tank and submerged $2 \mathrm{~cm}$ below the water level in the tank. This helps in concealing the location of the hidden platform in the water tank. The quadrant for the release of the study animals into the water tank and site for the location of the escape platform were maintained throughout this study. The release of the rats into the tank of water was carried out with the animals rear coming in contact with the water first, to relieve the animals of the anxiety of drowning-like effect.

The rats were timed for the duration taken to find a hidden escape platform. The time obtained appropriately reflect the latency time record (Alvin and Terry, 2009). The Probe time record was the duration taken by the rats in the quadrant that used to house the escape platform after it was removed, within a 60 seconds period- a measure of memory. These protocol of trainings were adapted from earlier studies (Morris et al, 1982; Sutherland et al, 1983).

\section{Evaluating Memory through Morris Water Maze} (MWM) Task

Evaluation of memory through the MWM essentially relies on its probe trial time protocol. This protocol evaluates the duration of time spent in a quadrant that formerly housed an escape platform by animals that had previously identified that escape platform correctly, as a demonstration of memory retention for that location. Hence, suggesting that the animals have developed a spatial bias and memory for the escape quadrant (Morris et al, 1982; Sutherland et al, 1983).

\section{Evaluating Learning through Morris Water Maze Task}

The learning aspect in the Morris water maze task was evaluated by observing the trend of the numerous records of the latency time that it takes the rat to locate the hidden platform during the training sessions. A decreasing trend in the duration of time needed to locate the hidden platform following subsequent trials in the water maze task suggests learning had occurred.A consistently decreasing latency-time with any new session of water maze task suggest consistent learning.

\section{Evaluating asymptomatic level of performance} (ALOP) in Morris Water Maze Task

Asymptomatic level of performance was identified as the point at which it takes the rats 10-15 seconds for four consective trials to locate the hidden platform in the water maze tank (Alvin and Terry 2009).

\section{Tissue preparation}

At the end of the study period, the rats were anaesthetized with an inhalational anaesthetic agent called chloroform. The rats were placed into an improvised fume-box individually, after which the box was sealed for 2-4minutes with the anesthetic agent in situ, to ensure adequate anaesthetisia. Each of the anaesthetizedsubject was then removed from the box and decapitated and the decapitated head was immediately fixed in $10 \%$ formalin. The head of ratswas then dissected to remove the brainsfor assessment. The brain was sectioned and the hippocampal region was mounted on a slide after embedding onto paraffin blocks. This mounted sections were stained using the Marsland and Glees techniques. Correspondingly similar regions of the section were visualized under the microscope at low and high magnifications and assessed for histological changes or similarities.

\section{Statistical analysis}

Statistical analyses were conducted using the statistical package for social sciences for windows (SPSS, version 15, SPSS Inc., UK). A tabular presentation of the percentage equivalence of the probe time scores for each of the groups was made. Independent t-test for the statistical difference of the probe time across the different study groups was assessed. Furthermore, one way analysis of variance (ANOVA) was carried out to assess the difference of the probe time in relation to gender and past history of exposure to cigarette smoke. Repeated measure analysis of variance was also carried out for the latency time records obtained in the study. The initial latency time records of the rats was used as a covariate to minimize any inter animal variation that may modify the performance of the animals. This repeated ANOVA was to ascertain the contribution of the gender and past history of exposure to cigarette smoke on the learning ability of the rats. Finally, the mean latency time records for each session of the water maze task was also graphically presented to clearly demonstrate the point at which asymptomatic level of performance was achieved. The asymptoatic level of performance corresponds to the point where the latency time is between $10-14$ seconds for the subsequent 4 consecutive task. The level of significance for all these statistical assessments was taken as $P \leq 0.05$.

\section{RESULTS}

Figure 1 represent a graphical illustration of the latency time for locating the hidden platform in the water tank, recorded for each sessions of the water maze task for the rats in group $C$, i.e. male exposed group.. The graphs illustrated that there was a decreasing trend in the latency time records with each new session of water maze task. Furthermore, the figure displays that the point of ALOP for all the rats in this group was firmly established at the 4th session and that at this point of ALOP the corresponding graphical outlines for all the rats essentially aligned together into a single outline. Hence implying that after the point of ALOP, the time spent in searching for the hidden platform-learning was predictable and consistent in all the rats in this group. 


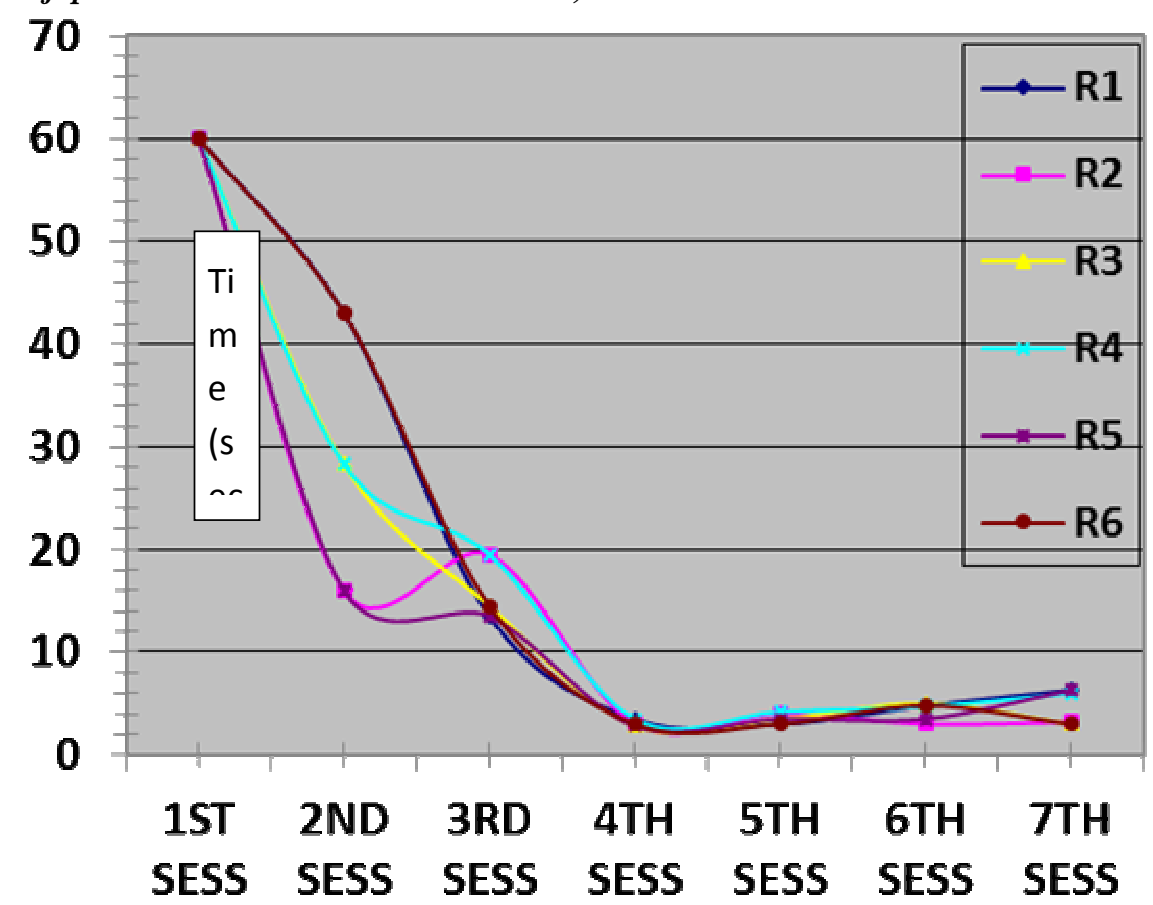

Figure 1: The latency time score of male exposed rats for each session of the water maze task

Figure 2, is a graphical illustration of the latency time recorded for each sessions of the water maze task foreach subject in group D-, female exposed group, in locating the hidden platform in the water tank. The figure (2) depicted that there was a decreasing trend of the latency time records with each new session of water maze task. However, there appears to be greater variability in the trend of the decrease in latency time (represented by the steepness in the outline) for these group compared to that observed for the male exposed rats (i.e. group C) in figure 1 . The variability in the steepness of the decrease in the latency time with successive session of water maze task was displayed by the non- alignment of the illustrative outlines representing the latency time records for each animal throughout the sessions as illustrated in figure 2.Furthermore, one of the six outlines in Figure 2revealed that ALOP was attained earlier (3rd session) by one of the rats, however, the average ALOP for all the rats in this group was firmly established by the 4th session. Suggesting that consistency and ease of learning for rats in this group seems to be slightly lower than that of the male exposed rats as displayed in figure 1.

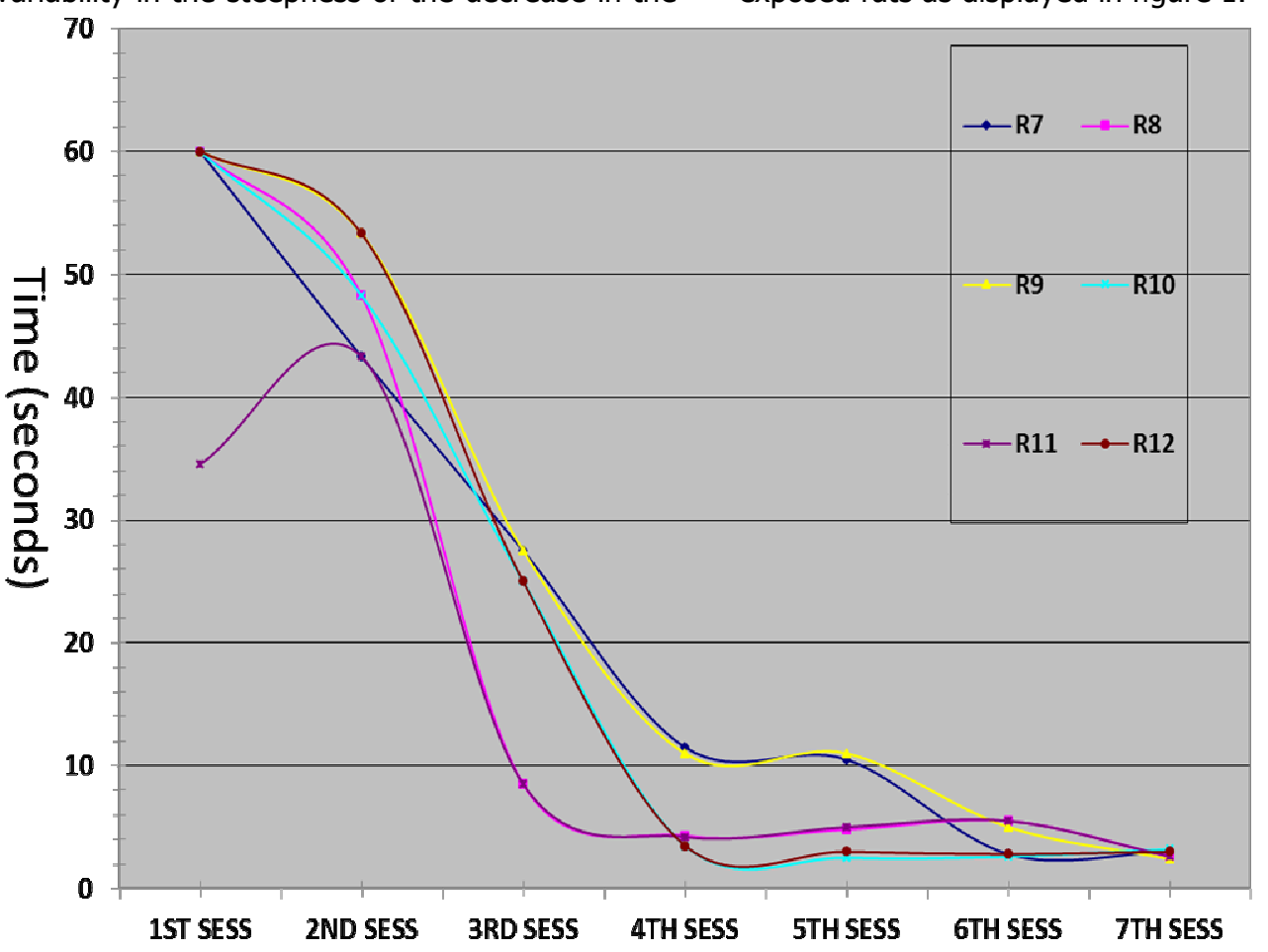

Figure 2: The latency time score of female exposed rats for each session of maze 
Bajopas Volume 8 Number 2 December, 2015

Figures 3 is a graphical illustration of the latency time records for the individual rats in group $A$ (control male) with ALOP firmly attained by the fifth session of the water maze task. Even though, the earliest attainment of the ALOP was noted by the 3rd session of water maze task in 4 rats belonging to this group. However, at the 4th session, the latency time for two (2) of the rats increased above the preceding latency time records observed at the 3rd session. Thereby, abolishing the initial trend of steep decline in latency time with each new session of water maze task, hence negating the definition of ALOP. The same pattern of abolishing the steep decline in latency time with each new session of water maze task was revealed at the 6th session. However, this reversal of decline observed at the $6^{\text {th }}$ session was still within the defined time durations for ALOP (10-14 seconds). This pattern of decline in the magnitude of the latency time records for most of the rats in this group seemed haphazard, less steep and highly variable compared to those observed for the preceding study groups (i.e. exposed rats). Hence implying that the actual learning process within a population of seemingly normal subjects may be highly variable.

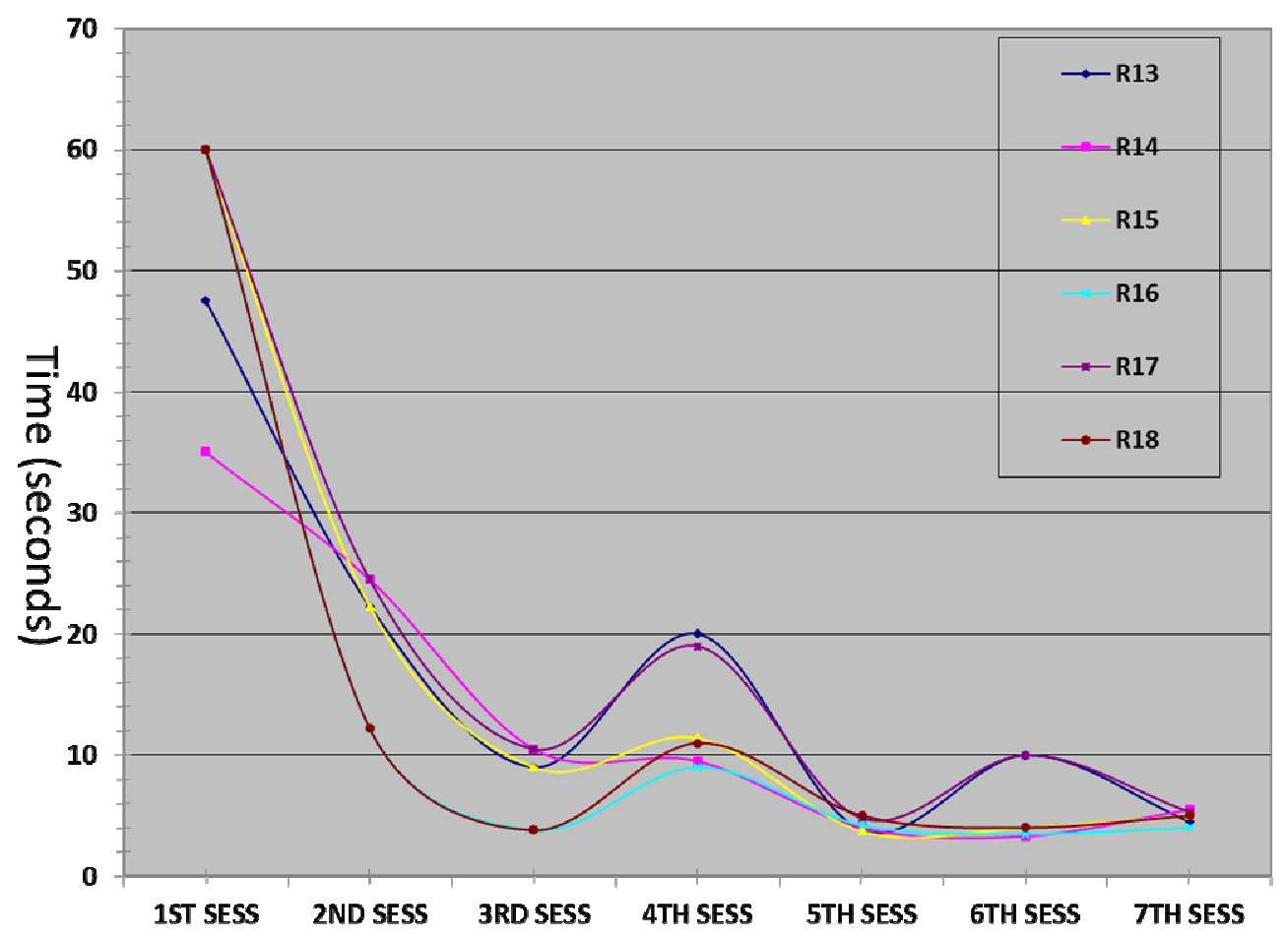

Figure 3: The latency time score of male controlrats for each session of maze task

Figures 4 revealed the latency time records for the individual rats in group $B$ (control female) and even though ALOP was attained earlier- 4th session for a few rats in this group, the average ALOP for this group was firmly attained by the 5 th session of the water maze task. Furthermore, the steepness of decline in the magnitude of latency time with successive session of water maze task was highly obliterated for a number of rats at the 3rd session and subsequently re-established by the $4^{\text {th }}$ session. Depicting a large variability in the consistency of learning within this supposedly unaffected group of rat. 


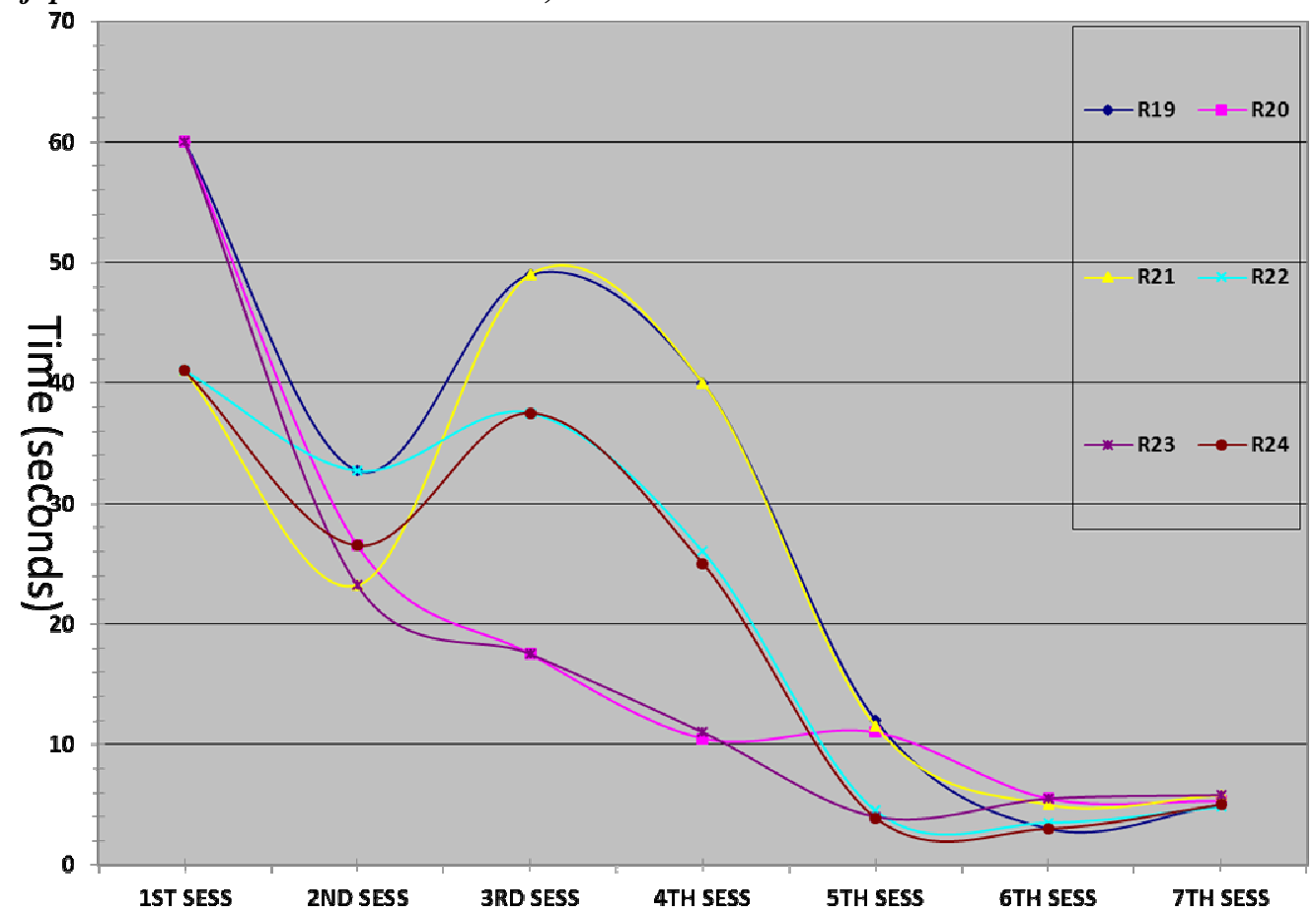

Figure 4: The latency time score of female controlrats for each session of maze task

Table 1 illustrates the percentage averaged probe time recorded for each of the study groups in the water maze task. A percentage above $25 \%$, implied that the search for the hidden platform within the search time (usually 60seconds) was not due to chance. Hence, the outcome of this study that revealed an average probe time for the study participants of $30 \%$ and above, implies that these current findings cannot be attributed to chance.

Table 1: Gender variation in percentage probe time within 60 seconds test duration.

\begin{tabular}{ll} 
Groups & Percentage time \\
\hline $\begin{array}{l}\text { Male,Control(group A) } \\
n=6\end{array}$ & $33.6 \%$ \\
$\begin{array}{c}\text { Male,Exposed (group C) } \\
n=6\end{array}$ & $36.1 \%$ \\
$\begin{array}{c}\text { Female,Control(group B) } \\
n=6\end{array}$ & $31.1 \%$ \\
$\begin{array}{c}\text { Female,Exposed (group D) } \\
n=6\end{array}$ & $35.0 \%$ \\
\hline of Wistar rats in each of the groups A, B,C and D respectively.
\end{tabular}

Table 2 displays the result of independent $t$ - test for the probe time records in relation to the rats (regardless of gender discrimination) history of exposure to cigarette smoke. The outcome of this study indicated that there wasno significant difference between the probe score of the exposed and nonexposed rats $\left(t_{22}=1.62, P=0.12\right)$. However, the exposed groups of animal had a longer duration of stay (21.08seconds) in the target quadrant compared to the control groups. Hence, suggesting that the memory for the location of the hidden platform was more in the exposed rats compared to the nonexposed, however, the difference was not significant.

Table 2: Differences in probe time between exposed and non-exposed rats.

\begin{tabular}{|c|c|c|c|}
\hline Group & $\begin{array}{l}\text { Mean probe time } \\
\text { (seconds) } \pm \\
\text { deviation }\end{array}$ & $\mathrm{T}$ & $\mathrm{P}$ \\
\hline $\begin{array}{c}\text { Exposed } \\
n=12\end{array}$ & $21.08 \pm 2.61$ & 1.62 & 0.12 \\
\hline $\begin{array}{l}\text { Non-exposed } \\
n=12\end{array}$ & $19.42 \pm 2.39$ & & \\
\hline
\end{tabular}

statistical significance assumed at $\mathrm{P} \leq 0.05$. $\mathrm{n}=$ number of rats in the group 
Bajopas Volume 8 Number 2 December, 2015

Table 3,shows the result of one way analysis variance for the probe time records according to the history of exposure to smoke and gender. Findings from this study shows that there was an insignificant difference in probe time across gender $\left(F_{2,15}=1.196, P=0.33\right)$. This result suggest that the phenomenon of gender plays little or no role in the memorization of the quadrant that housed the hidden platform earlier. However a closer look at the duration of time spent in the target quadrant revealed that the male rats spent more time than the female rats.

Table 3: Variation in probe time across gender and exposure characteristic to cigarette smoke.

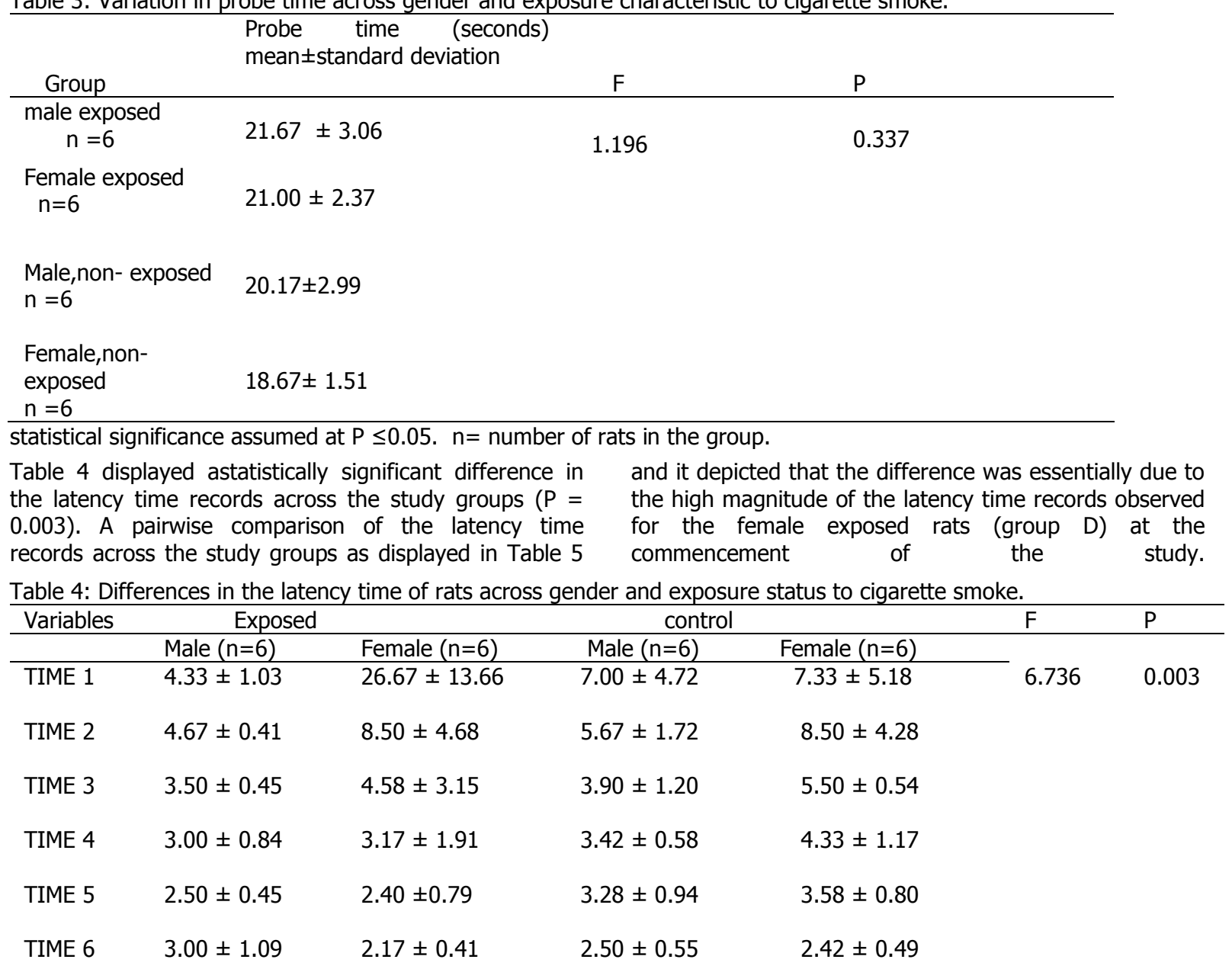

Statistical significance assumed at $\mathrm{P} \leq 0.05$. $\mathrm{n}=$ number of rats in the group.

Table 5: Pairwise comparison of latency time revealing differences across the study groups.

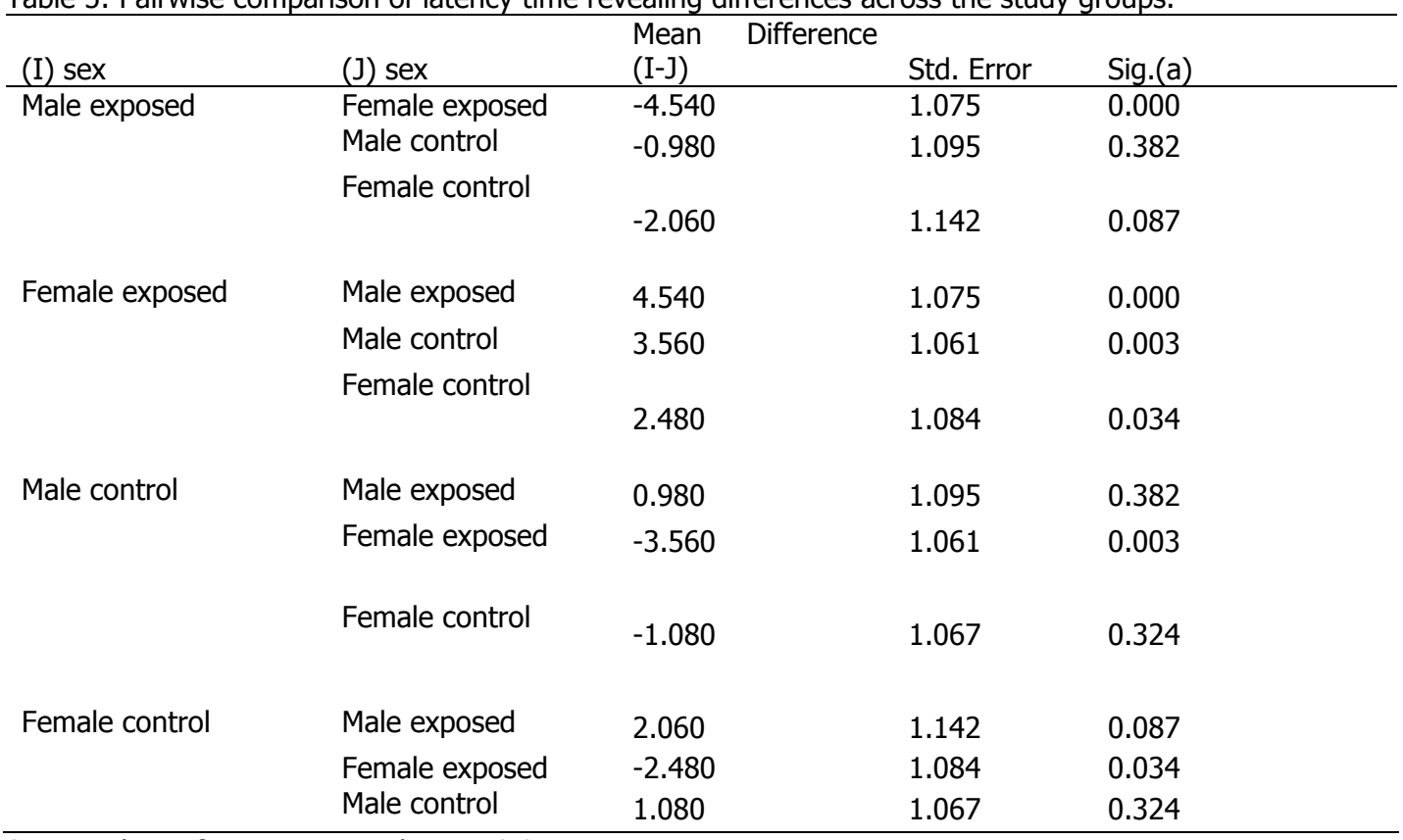

Statistical significance assumed at $\mathrm{P} \leq 0.05$ 
Bajopas Volume 8 Number 2 December, 2015

\section{Tissue sections}

Marsland and Glees stained section for the hippocampus of the non-exposed rats revealed a general microscopic similarity between the males and female section. In addition, the microscopic findings of the exposed group of rats were also similar for both male and female exposed rats. Hence the structural appearance of the section was independent of gender.

The cornuammonis 1 and 2 (CA1, and CA2) regions of the hippocampus revealed a pattern of widely

interspersed pyramidal cells with numerous intercellular spaces- edematous changes in the exposed groups of rats. Furthermore, the pyramidal cells revealed a highly condensed and pigmented nucleus. These microscopic changes are evident in the sections of the hippocampus from the exposed rats at a high magnification of four hundred (x400x) as illustrated in Figure 2.The general outline of the hippocampus of the exposed rats at a lower magnification of sixteen (16) was also illustrated in Figure2.

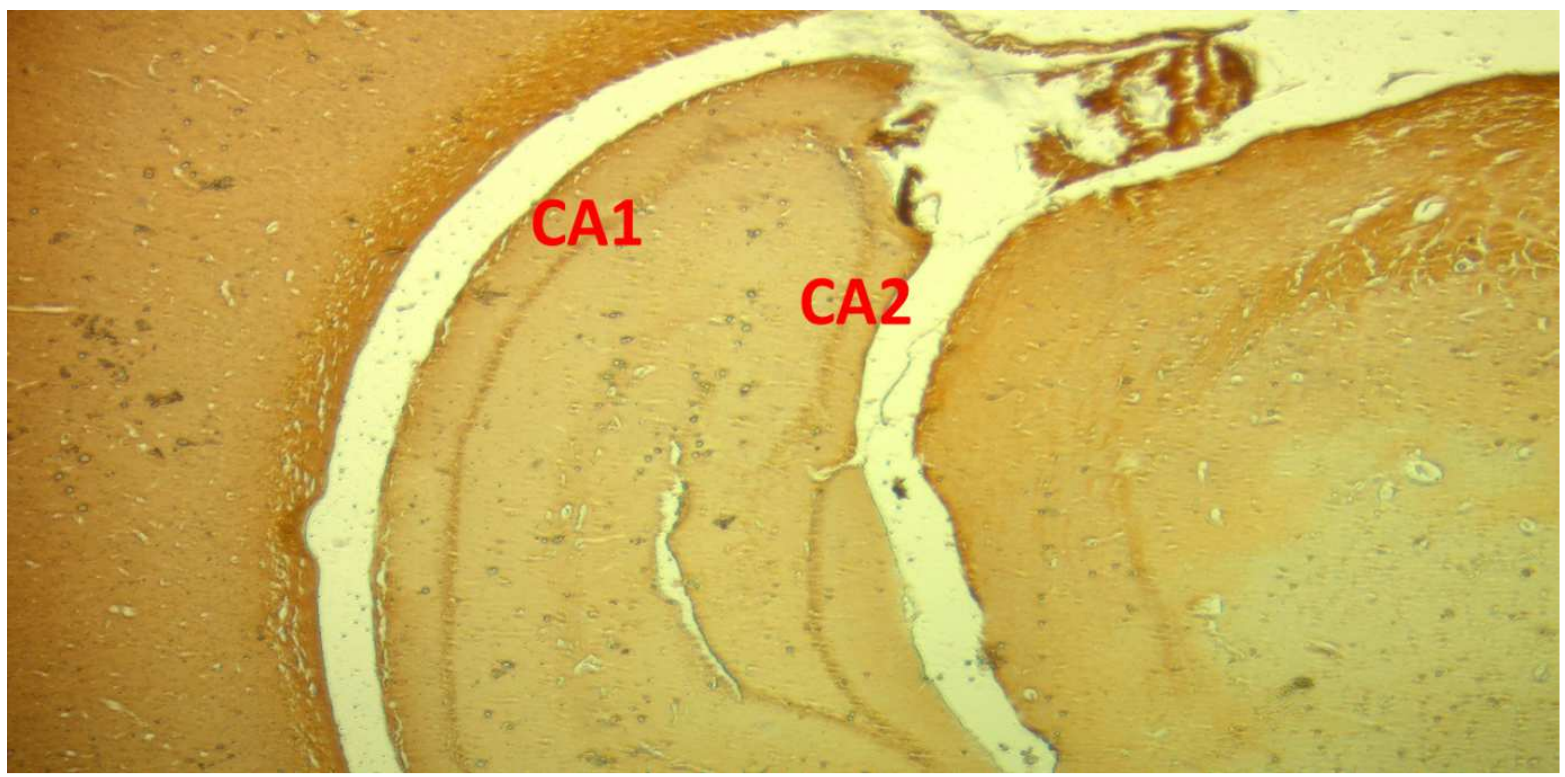

Plate 1: Hippocampus of the non-exposed rats (Marsland and Glees xl6). CAl and CA2 = Cornu ammonis 1 and 2

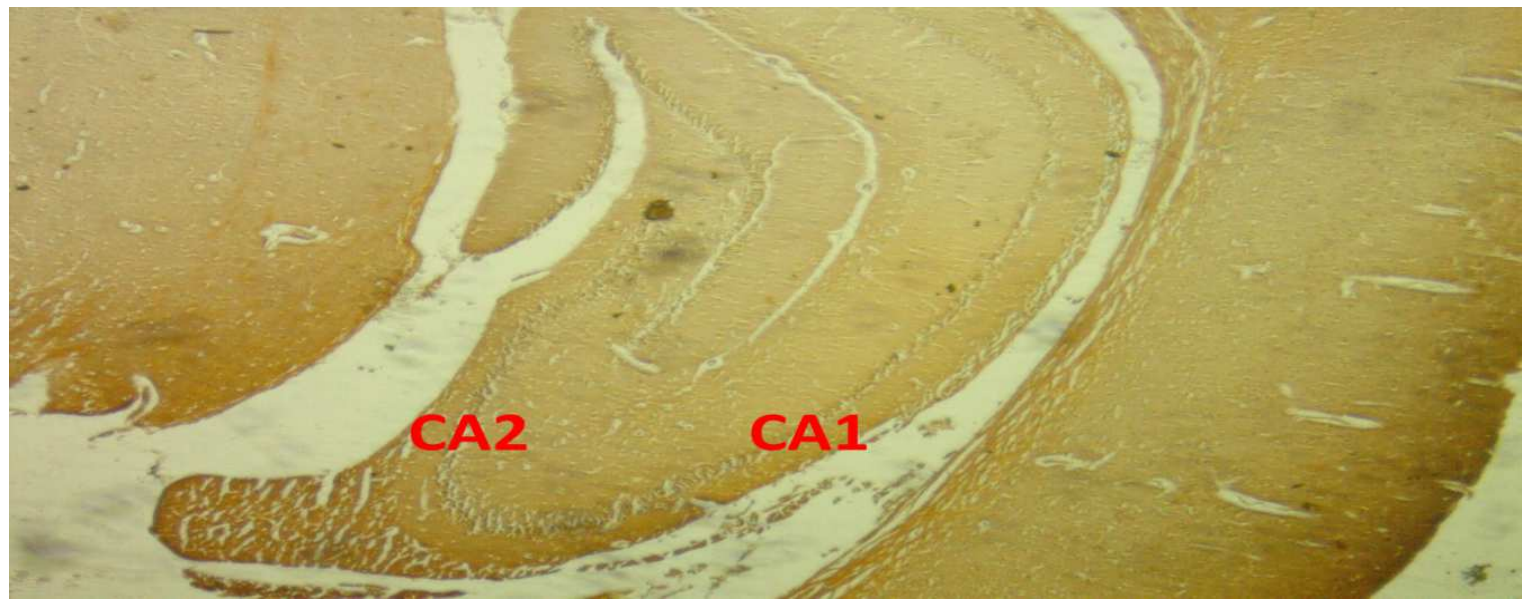

Plate 2: Hippocampus of the exposed-rats (Marsland and Glees x16). CA1 and CA2 = Cornu ammonis 1 and 2 


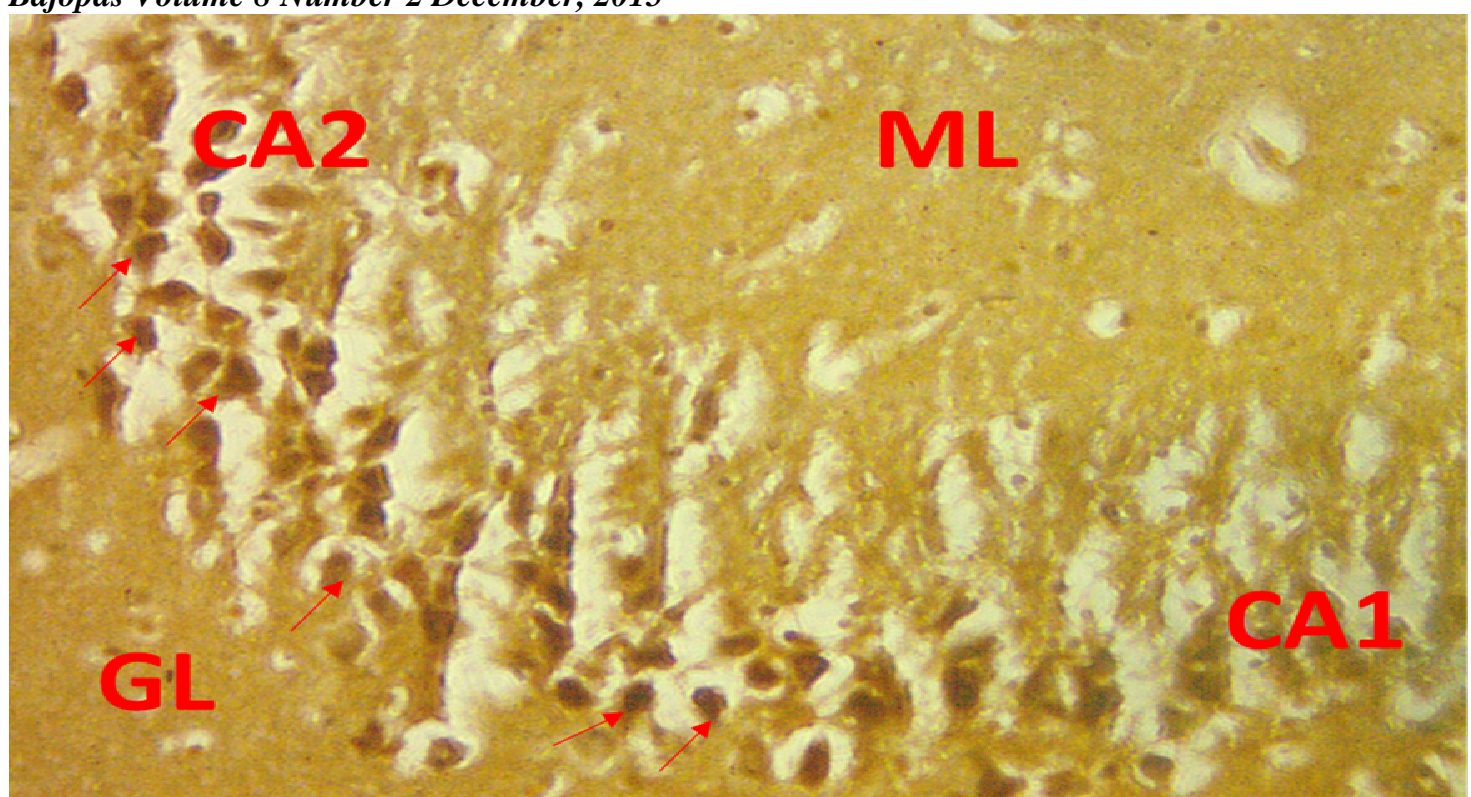

Plate 3: Hippocampus of the exposed rats (Marsland and Glees $\mathrm{x} 400$ ). CA1 and CA2 = Cornu ammonis 1 and 2, ML=molecular layer of hippocampus, $\mathrm{GL}=$ granular layer of hippocampus, short arrows $\longrightarrow$ pointing at hyperpigmented-nucleus of pyramidal cells

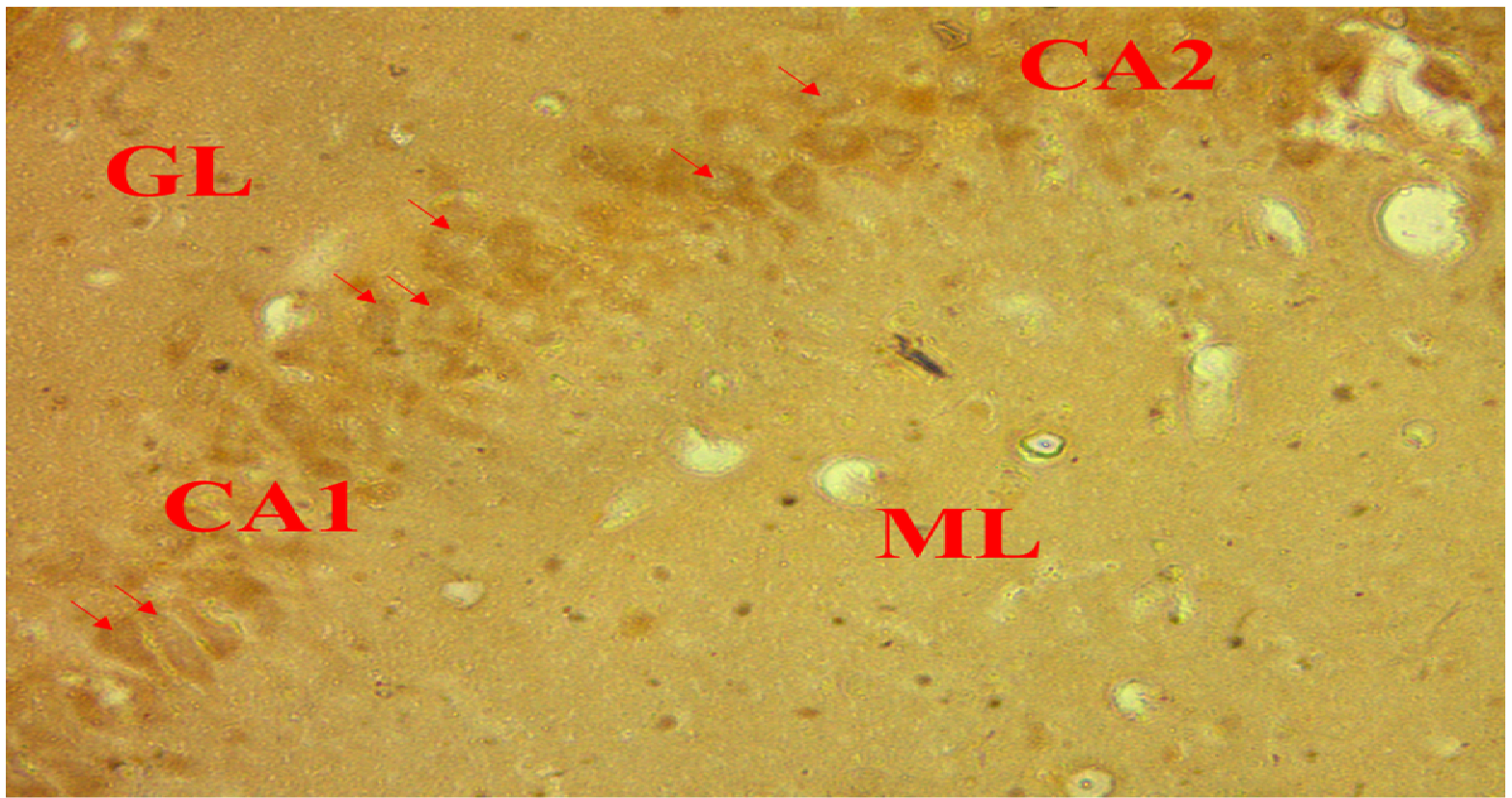

Plate 4: Hippocampus of the non-exposed rats (Marsland and Glees x400). CA1 and CA2 = Cornu ammonis 1 and 2, ML=molecular layer of hippocampus, GL= granular layer of hippocampus, short arrows $(\longrightarrow$ pointing at euchromatic-nucleus of pyramidal cells

Furthermore, the sections of the non-exposed rats at higher magnification revealed that the nucleus of the pyramidal cells in the hippocampus including the CA1 and $C A 2$ regions were not hyper pigmented. And the pyramidal cells were not excessively spread-out into numerous layers of row (dispersion). Furthermore there was little or absent interstitial edematous changes. This microscopic features of the nonexposed rats are illustrated in Figure 4. An illustration of a section of non-exposed rats-hippocampus at a magnification of sixteen (x16) was illustrated in Figure 1 in assessing the structural changes in the exposed groups of rats.

\section{DISCUSSION}

Although there were some inter-individual differences regarding achievement of ALOP for different subjects in different study groups, suggesting that some study rats are innately better task-performers than others. Nevertheless, the exposed and non-exposed groups of rats averagely attained ALOP by the $4^{\text {th }}$ and $5^{\text {th }}$ session of water maze task, regardless of gender.. In addition, this study revealed that learning in the form of locating a hidden platform in a water maze was accomplished and attained by all subjects. However, the learning pattern was more consistent and seemed easier among the exposed-males than in the exposedfemales. Similarly, the pattern of learning was also seemed easier for the control-males than in the control-females. 
Bajopas Volume 8 Number 2 December, 2015

These learning patterns were assessed in relation to the steepness and consistency of the decline in latency time with each session of water maze task. Hence, the female-exposed rats seemed to have the most of difficulty in learning the water maze task (nevertheless learning was established), while the male-exposed had the least difficulty at learning.

The assessment of memory-measures for the femaleexposed rats revealed a higher level $(35.0 \%)$ compared to those of the female-controls (31.1\%). A similar trend was observed for the male study groups, with the control and exposure groups having 33.6\% and $36.1 \%$ respectively. In addition, the male groupboth exposure and non-exposed have a higher memory level than their corresponding female groups (exposed and non-exposed groups). Hence, the current study suggest that the memory capacity and hence ability to retain and recall information about a learned event is highest among the exposed group of rats with males better than females. These findings as in this study are potentially of immense significance especially on the capabilities of learning, ALOP- a measure of learning and memory for tasks following cessation of long term-CSE.

Furthermore, in this study, assessment of the structural changes in the hippocampus of study rats was undertaken. The microscopic changes of increased condensation (pyknosis) of nuclei, edematous changes and disruption of the fine linear architecture of the pyramidal cells of the hippocampus especially in the cornuammonis 1 and 2 regions (CA1 and CA2) of the exposed rats were evident. This microscopic structural changes were similar in both the female and male CSE rats. Therefore, the findings from this study on functioning in learning, ALOP and attainment of memory for the exposed groups of rats ( showing changes -pyknosis, edema, architectural disruption) at a comparable rate to those of nonexposed rats is remarkable. Especially because this structural findings suggest that either this hippocampal changes are beneficial effects of longterm CSE and a concomitant cessation of CSE as in the setting of this study. Or the structural changes are detrimental and necessitated the study subjects to enlist a different and bettermechanism in performing these functions of learning, ALOP and memory. However, the current finding that female-exposed rats had the highest difficulty in learning the maze task (no prejudice to the fact that it also attained learning for the task) is very revealing. Especially against the backdrop of high and comparable level of memorization and ALOP for this same task, exhibited by this same female-exposed rats. This suggest that either the female apparatuses for learning seemed be slowest at benefiting or it is worst affected by the CSE and hence exhibiting a slower recovery. The female gender is a probable reason for this effect. Therefore the issue of whether the exposed group of rats enlist the function of a different and more efficient apparatus to perform these functions seems highly unlikely.

The better improvement in performance of the maleexposed rats in learning, ALOP and memorization as observed in this study supports the suggestion that the study settings had beneficial effects on the performance of this function In-addition this beneficial effect seems to be dependent on the duration of cessation CSE rather than the long term CSE. Especially because, long-term CSE has earlier been shown to foster detrimental effects on learning (Taofik, 2013). Hence reducing the possibility of the potential suggestion that the study setting probably resulted in the enlisting of other apparatus for this function. Therefore, the current study reveals that the detrimental effect of CSE is most deleterious in the female CSE groups and cessation of CSE alleviates this effect rapidly in male compared to the females, however both gender benefit immensely from the cessation of CSE.

The findings from this current study are in keeping with earlier findings in the literature, in which the performance of un-impaired male Wistar rats in memory task had been revealed to be better than that of an un-impaired female rats (Cimadevillaet. al., 2004). Which is similar to the current finding of a higher memory measure in the control male $(33.6 \%)$ compared to the control female $(31.1 \%)$. This gender-based higher performance in males is also replicated in the CSE group., with the memory measure for the exposed male $(36.1 \%)$ higher than those the exposed-female (35\%). The learning (for locating a hidden platform) in this study also reveals a gender-based higher performance for the males (both exposed and non-exposed) over and above that of female (exposed and non-exposed).

In addition, another measure of learning called ALOP was explored in this study. ALOP was defined as the ability of un-impaired (control) rats to identify hidden platform in the water maze within $10-15$ secs and continue to locate it within this time span for another 4 consecutive trials in a 10-20 trials protocol. Furthermore, it was revealed that achieving ALOP usually required a few days of MWM task (Slotkinet. al., 2006). In the current study, ALOP was essentially attained earlier for the exposed groups-4th session, than for the control group-5th session. However, many of the rats in the control-male group and few rats in the control and exposed female groups attained the ALOP earlier suggesting an interindividual differences. Hence, the finding of the current study regarding the attainment of ALOP essentially reveals that all the study groups attained it.

The histological changes observed in the microscopic slides of the hippocampus of these study animals revealed that there was a distinct dichotomy between the sections from the no-exposed and those of the exposed groups. However, there was no discernable difference in the microscopic anatomy that relates to a difference in gender. Furthermore, the presence of a distorted architecture of the pyramidal cell layer, the condensation and pyknosis of the nuclei of the pyramidal cells and the edematous vacuoles of the brain tissue all lend credence to the effects of CSE, because this changes were absent in the control groups.

Furthermore, the role of these structural changesespecially edema in the functions of the brain can predictively be envisaged as detrimental which is in keeping with the functional deficit observed in CSE mice by Taofik, (2013). 
However, the findings from the current study suggest that the function of the memory and learning apparatuses wee functionally salvaged and improved upon which is in keeping with the findings of Hillary et. al., (2003).

The study of Hillary et al.,(2003) revealed that in the presence of gross structural changes that may inhibit adequate functioning of the brain, the phenomenon of spacing and those of repetition may improve the brain functioning (Hillary et. al., 2003). Hence, it is probable that the repetition of the water maze task over specific time intervals had immensely contributed to the enhancement of learning and memory. This is probably due to the fact that when a conditioned task (removing the rats from the water when it locates the hidden platform) is coupled with an unconditioned activity (swimming to identify the hidden platform), it propels the accomplishment of the unconditioned activity. In addition, spacing phenomenon which essentially involves allowing for a period of time between each task before the same task is repeated was also considered by Hillary et al., (2003). And during this inter trial periods, no additional trial or task are carried out. Furthermore, spacing phenomenon has also been shown to promote memory development even in the setting of a Clinical implications of this study.

The empirical and epidemiological information of general improvement in health status and functioning that are utilized in counselling CSE patients on the need for them to stop CSE seems to have been replicated for the learning, ALOP and memory functions of the brain by this current study. Hence

\section{REFERENCES}

Ahmadnia, H., Mohsen, G., Mohammad, R.M., and Khaje-Dalouee, M.(2007). Effect of cigarette smoke on spermatogenesis in rats.J Urol,4(3).

Alvin, V.,andTerry, Jr. (2009). Spatial Navigation (Water Maze) Task. In: Buccafusco JJ, editor. Methods of Behavior Analysis in Neuroscience, 2nd edn. Boca Raton (FL): CRC Press, Chap 13.

Bliss, T.V.P., and Collingridge, G.L. (1993). A synaptic model of memory: long- term potentiation in the hippocampus. Nature,361,31-39.

Brandeis, R., Brandys, Y., andYehuda, S. (1989). The use of the Morris Water Maze in the Study of memory and learning. Int J Neurosci,48, 2969.

Cervilla, J.A., Prince, M., andMann, A. (2000). Smoking, drinking, and incident cognitive impairment: a cohort community based study included in the Gospel Oak project. $J$ Neurol Neurosurg Psychiatry, 68,622-6.

Cimadevilla J.M., Fenton, A.A., and Bures, J.(2004). Continuous place avoidance task reveals differences in spatial navigation in male and female rats. Behav Brain Res,107,161-9.

Elwood, P.C., Gallacher, J.E., Hopkinson, C.A., Pickering, J., Rabbitt, P., Stollery, B., Brayne, C., Huppert, F.A., and Bayer, A. (1999). Smoking, drinking and other lifestyle factors moderate to severe brain damage (Hillaryet. al., 2003). Hence, in this current study, the period of time between on trial and the next trial may imply 'spacing' with its associated beneficial effect.

\section{Conclusion}

This study has revealed that intermittent repeated task performance following cessation of long term CSE was associated with the both maintenance and improvement of learning and memory in generally. Furthermore, the males performed better than the females and the exposed rats performed better than the non-exposed in ALOP and memory. However, there was some difficulty in learning experienced by the female-exposed rats(though learning occurred) compared ease of learning for the other study groups. In addition, the current study revealed that ALOP was firmly attained earlier (4th session) for all the rats in the exposed groups compared to those of all the rats of the control groups (5th session). Hence, these findings imply that there was no detrimental effect on learning, memory and ALOP following intermittent repeated task performanceand cessation of long term CSE. Hence, the cessation of CSE in the setting of repetitive trials of water maze task seemed to ensure that any potential deleterious effect of long-term CSE was attenuated.

implying a clinical relevance for this finding for evidence based data.

and cognitive function in men in the Caerphily cohort. J Epidemiol. Communty Health, 53, 9-14.

Ernst, M., Heishman, S.J., Spurgeon, L., and London, E.D. (2001). Smoking history and nicotine effects on cognitive performance. Neuropsychopharmacology, 25, 313-319.

Ford, A.B., Mefrouche, Z., Friedland, R.P., and Debanne, S.M. (1996). Smoking and cognitive impairement: Apopulation- based study. J Am GeriatrSoc, 44, 905-906.

Fried, P.A., Watkinson, B., andGray, R. (2006). Neurocognitive consequences of cigarette smoking in young adults - a comparison with pre-drug performance.Neurotoxicology and Teratology, 28, 517-525.

Galanis, D.J.,Petrovich, H., Launer, L.J., Harris, T.B., Foley, D.J., and White, L.R (1997). Smoking history in middle age and subsequent cognitive performance in elderly JapaneseAmerican men. The Honolulu Asia Aging Study. Am J Epidemiol., 145, 507-515.

Giampaolo, M., Cammalleri, M., Luigia, T., Francesconi,W., Saba, P., Maria, T., Andrea, V., andVincenzo, C. (2000). Prenatal exposure to the low concentration of Carbonmonxide disrupts Hippocampal LTP in rat offspring. $J$ Pharmcol Exp Therapeut, 244, 728-734. 
Hillary, F.G., Schultheis, M.T., Challis, B.H., Millis, S.R., Carnevale, G.J., and Galshi T,DeLuca, J. (2003). Spacing of repetitions improves learning and memory after moderate and severe TBI.Journal of Clinical and Experimental Neuropsychology,25, 4958Kalmijn, S., van Boxtel, M.P., Verschuren, M.W., Jolles, J., andLauner,

L.J. (2002). Cigarettesmoking and alcohol consumption in relation to cognitive performance in middle age. Am J Epidemiol, 156, 936-44.

Liu, J.T., Lee, I.H., Wang, C.H., Chen, K.C., Lee, C.I., and Yang Y.K. (2013). Cigarette smoking might impair memory and sleep quality. Journal of the Formosan MedicalAssociation, $112,287-290$

Lynch, M.A. (2004). Long Term Potentiation and Memory. Physiol Rev,84,87-136.

Mendrek, A., Monterosso, J., Simon, S.L., Jarvik, M., Brody, A., Olmstead, R., Domier, C.P., Cohen, M.S., Ernst, M., and London, E.D. (2005). Working memory in cigarette smokers: Comparison to non-smokers and effects of abstinence. Addict Behav, 31(5), 833-844.

Morris, R.G.M., Garrud, P., Rawlins, J.N., and Keefe, J.O. (1982). Place navigation impaired inrats with hippocampal lesion. Nature,297, 681683.

Morris, R.G.M. (1984). Development of a water-maze procedure for studying spatial learning in the rat. J Neurosci. Methods,11, 47-60.

Nowakowska, E., Kus, K., Florek, E., Czubak, A., andJodynis-Liebert, J.(2006). The influence of tobacco smoke and nicotine on antidepressant and memory-improving effects of venlafaxine. Hum Exp Toxicol,25,199-209.

Eser, O., Cosar, M., Sahin, O., Mollaoglu, H., Sezer, M., Yaman, M.,andSongur, A. (2007).The neuroprotective effects of caffeic acid phenethyl ester (CAPE) in the hippocampal formation of cigarette smoke exposed rabbits. Neurosurg Path, 39, 433-7
Schinka, J.A., Belanger, H., Mortimer, J.A., and Borenstein, G.A. (2003). Effects of the use ofalcohol and cigarette on cognition in elderly African American adults.J IntNeuropsychol.Soc, 9, 690-697.

Slotkin, T.A., Pinkerton, K.E., and Seidler, F.J. (2006). Perinatal environmental tobacco smoke exposure in rhesus monkeys: critical periods and regional selectivity for effects on brain cell development and lipid peroxidation. Environmental Health Perspectives,114, 349.

Sutherland, R.J., Whishaw, I.Q., and Kolb, B.A. (1983). Behavioural analysis of spatial localization following electrolytic, kainite- or colchicines- induced damage to the hippocampal formation in the rat. Behav Brain Res, 7, 133-153.

Taofik, O. (2013). Effects of chemical compounds found in cigarette smoke on cognitive ability of mice. A thesis submitted in partial fulfillment of the requirements for a baccalaureate degree in Biology in cursu honorum.Submitted to: The Honors Program, Saint Peter's University.

Toumane, A., andDurkin, T.P. (1993). Time gradient for post-test vulnerability to scopolamineinduced amnesia following the initial acquisition session of a spatial reference memory test in mice. Behav Neural Biol, 60, 139.

Yolton, K., Dietrich, K., Auinger, P., Lanphear, B.P., and Hornung, R. (2005). Exposure to environmental tobacco smoke and cognitive Abilities among U.S. children and adolescents, Environ. Health Perspect, 113, $98-103$. 